FORMATION Formation emploi

Revue française de sciences sociales

96 | octobre-décembre 2006

Quand la qualification fait débat(s)

\title{
Le travail face à la restructuration productive : le cas d'un centre d'appels
}

The productive restructuring of work: the case of a call centre

Erwerbsformen im Wandel: am Beispiel der Call-Center

José Calderón

\section{OpenEdition}

Journals

Édition électronique

URL : http://journals.openedition.org/formationemploi/2509

DOI : 10.4000/formationemploi.2509

ISSN : 2107-0946

Éditeur

La Documentation française

Édition imprimée

Date de publication : 1 octobre 2006

Pagination : 11-24

ISSN : 0759-6340

Référence électronique

José Calderón, «Le travail face à la restructuration productive : le cas d'un centre d'appels », Formation emploi [En ligne], 96 | octobre-décembre 2006, mis en ligne le 14 décembre 2009, consulté le 30 octobre 2020. URL : http://journals.openedition.org/formationemploi/2509 ; DOI : https://doi.org/ 10.4000/formationemploi.2509

(c) Tous droits réservés 
plus larges du monde du travail qui modifient la relation des travailleurs à leur travail et qu'il convient de questionner.

Dans les organisations tayloriennes, on laisse peu de place à l'improvisation. L'industrie produit en masse des biens standardisés et l'activité de production est largement planifiée. La direction prétend prescrire entièrement les activités de travail et traiter les salariés comme de simples exécutants. Les rivalités de classe s'organisent alors autour de l'usage objectif du temps de travail, c'est-à-dire autour de l'intensité du travail objectivé et rationalisé par les bureaux de méthodes ${ }^{1}$, puisque le temps subjectif, l'activité réelle des salariés et leurs motivations subjectives pour transgresser les normes, est tout simplement nié par l'ingénierie de la «meilleure et seule façon de produire" (one best way) (Abécassis et Roche, 2001)2.

La tendance aujourd'hui est d'une autre nature. Dans la nouvelle organisation, la foi ingénieriste dans la rationalisation totale des moyens cède la place à l'impératif de l'adaptabilité. L'appel à l'implication, à l'initiative, à l'autonomie, à la responsabilité des salariés vise à combler l'incapacité des bureaux de production à prescrire entièrement les activités de travail (Flichy et Zarifian, 2003). Les objectifs des organisations dépendent de plus en plus de la capacité d'adaptation des travailleurs aux aléas de la production. Les évolutions de l'organisation du travail requièrent en somme d'intégrer l'intelligence et la personnalité des travailleurs.

\footnotetext{
${ }^{1}$ Refus du chronométrage, freinage, coulage et autres formes de sabotage cristallisent alors cette opposition. Voir à ce propos, Pierre Dubois, Le sabotage dans l'industrie, Calmann-Levy, Paris, 1976.

2 L'intégration de la subjectivité n'est pas prioritaire sous le taylorisme. La gestion taylorienne de la force de travail est fondée sur l'intensification productive, l'expropriation de la connaissance et la suppression de l'autonomie ouvrière. Ces politiques ont paradoxalement favorisé la naissance d'une subjectivité collective antagonique, et dans ce sens, le taylorisme comme mode de contrôle a été considéré par certains comme un véritable échec. Du point de vue technique, le taylorisme a favorisé la rétention d'information puisqu'il ne reconnaît pas la distance entre travail et réel (Bonazzi, 2002). La constitution d'une qualification informelle a pu être ensuite utilisée par l'ouvrier pour humaniser puis s'opposer à une rationalisation technique et organisationnelle du travail qui l'ampute de l'expression de son initiative et de son point de vue (Linhart, 2004).
}

Dans les centres d'appels, les responsables ne se préoccupent pas uniquement d'objectiver les temps et les gestes du travail (le temps objectif de travail); au contraire, ils semblent s'intéresser à l'implication du salarié, à sa subjectivité, afin que ce dernier fasse un usage de son temps de travail et de ses compétences le plus efficace possible.

L'aspect subjectif de la main-d'œuvre, sa composition qualitative, intervient lors des processus de sélection et de recrutement. À cet égard, signalons l'importance accordée aux "compétences sociales ", (cf. Callaghan et Thompson (2002); Marie Buscatto (2002); Selma Venco (2003); Andrea del Bono (2001)). La solidité psychologique et, bien sûr, la motivation, mais aussi d'autres aspects comme la personnalité du candidat, son énergie, l'enthousiasme supposé, le «feeling », les capacités relationnelles (le ton, le sens de la discussion) sont autant d'éléments appréciés par les responsables du recrutement.

Pour autant, toutes les « compétences sociales» ne sont pas en adéquation avec le développement de l'activité : cet usage du temps subjectif de travail qui préoccupe de plus en plus la direction doit servir les objectifs de l'entreprise. Ainsi, l'appel à l'implication des travailleurs s'organise avec la mise en place de dispositifs de contrôle de type normatif qui visent à standardiser et à rationaliser le comportement adéquat au travail, à partir d'un modèle prédéterminé $\mathrm{du}$ «bon conseiller». En centre d'appels, les « compétences sociales » des salariés deviennent en somme un outil mis à la disposition des responsables pour parvenir à leurs fins.

En situation de travail, le télé-conseiller est ainsi obligé d'arbitrer entre plusieurs systèmes de contrainte : entre les impératifs quantitatifs et qualitatifs de l'activité (par exemple : répondre au maximum d'appels, en un minimum de temps, mais avec une certaine «personnalisation de la relation»), mais aussi entre le respect des objectifs de l'entreprise et de ses propres exigences éthiques: qu'est-ce que personnaliser la relation? Qu'est-ce qu'une réponse de qualité ? Dans ce sens, nous postulons que l'usage des capacités cognitives et relationnelles en tant qu'outil pour la production du service, l'usage des « compétences sociales», devient l'espace d'un enjeu autour de la finalité du travail entre les dirigeants et les salariés, dans le cadre de ce que les nouveaux théoriciens du process de travail appelè- 
rent, dans leur critique de Braverman³, la «compétition pour le contrôle $»^{4}$.

Dans un premier temps, nous essayerons de montrer en quoi la rationalisation du process de production de services est nécessairement incomplète dans les centres d'appels. Notre étude de cas, qui porte sur une plateforme téléphonique (PHONING), montre qu'en effet, la rationalisation est confrontée à de nombreuses difficultés liées à la nature de l'activité « fourniture de services ». Dans les centres d'appels, la qualité du produit dépend directement de la façon de travailler des salariés, le travail de ceux-ci étant soumis immédiatement au jugement du client. Dans un deuxième temps, nous analyserons comment les responsables de PHONING entendent combler le décalage entre moyens et résultats du point de vue de l'aspect qualitatif de l'activité : pour ce faire, nous nous appuierons sur l'analyse des dispositifs de coaching dans l'entreprise. Dispositifs qui influent tant sur l'aspect répétitif que sur l'aspect réactif de l'activité et qui sont présentés comme des éléments clés de la «professionnalisation » des salariés. Enfin, nous nous intéresserons aux pratiques de travail des télé-conseillers, plus particulièrement à la manière dont ceux-ci s'adaptent et/ou résistent aux contraintes

\footnotetext{
${ }^{3}$ Pour Braverman, l'organisation scientifique du travail n'est pas une méthode de gestion comme une autre, ni une science générale du travail, mais un trait fondamental du process de travail capitaliste qui offre des méthodes de contrôle applicables à tous les niveaux technologiques. L'objectif du taylorisme est d'enlever au travailleur le pouvoir discrétionnaire qu'il détient sur son travail et de contrôler la façon dont chaque activité est effectuée dans la logique de l'accumulation du capital.

${ }^{4}$ La notion bravermanienne de contrôle fera l'objet de critiques ( $c f$. Edwards, 1979, Burawoy, 1979) car trop réductrice : ainsi, il n'est pas vrai que l'employeur impose sans fin ses exigences aux travailleurs. Les pratiques de contrôle s'inscrivent au contraire dans une compétition pour le contrôle du process de travail ; c'est dans cette compétition que les travailleurs peuvent trouver des mécanismes pour résister à la déqualification. On peut dès lors considérer que si le temps subjectif de production est l'objet d'une attention particulière de la part des entrepreneurs, il est en même temps l'espace de contestation de rationalités en dispute. Autrement dit, il peut y avoir un appel à l'implication des travailleurs, mais rien ne préfigure le sens de cette implication. Dans ce texte, il s'agira en somme de rapporter les pratiques des travailleurs à leurs préoccupations, à leurs situations quotidiennes ainsi qu'aux enjeux définis par la trajectoire de l'entreprise et par leur propre trajectoire. Il ne s'agira pas de montrer le réel du travail, en opposition au prescrit, mais d'indiquer le sens accordé par les travailleurs à ce réel qui est aujourd'hui reconnu par tous, y compris par les responsables de l'entreprise qui entendent l'utiliser pour atteindre leurs propres objectifs.
}

de productivité et de rentabilité - contraintes temporelles et morales induites par l'organisation du travail - en fonction de leur trajectoire et de leurs propres exigences éthiques. Ces pratiques pouvant être à la base de collectifs de travail renouvelés que nous tenterons d'identifier et de définir.

\section{Encadré 1 \\ Méthodologie de l'enquête}

Les résultats présentés ici proviennent d'une immersion de quatre mois réalisée dans un centre d'appels du secteur de la distribution énergétique, dans la couronne parisienne. L'observation in situ des situations de travail a été complétée par des entretiens semi-directifs (30) d'une heure et demie en moyenne auprès des cadres, des responsables de formation, des représentants syndicaux et des travailleurs de la plate-forme téléphonique. Le guide d'entretien a été construit dans l'objectif de saisir cinq dimensions principales: la description du travail et de son organisation, la nature et les situations vécues des rapports avec la hiérarchie et le client, la nature et les situations vécues des rapports avec les collègues, le parcours scolaire, personnel et professionnel et les perspectives (personnelles, professionnelles) souhaitées ou envisagées. Nous avons ensuite procédé à une analyse de contenu transversale appliquée à l'ensemble des entretiens et à une analyse structurale propre à chaque entretien.

\section{PHONING AU COEUR DU «TOURNANT COMMERCIAL »}

PHONING est l'interface essentielle entre une entreprise encore publique et sa clientèle. Les salariés, pour la plupart des femmes, effectuent des tâches d'accueil, de gestion et de facturation. Elles travaillaient auparavant au service facturation. L'évolution rapide des technologies de l'information a nécessité le regroupement de ces agents sur les nouveaux espaces de travail. À leurs activités initiales 
s'est ajouté l'accueil téléphonique, tâche devenue prépondérante: il faut répondre aux appels des clients, les faire attendre le moins longtemps possible, prendre le maximum d'appels, leur proposer des services, traiter entièrement toutes les demandes, taper les conclusions commerciales des discussions. Les salariés sont également chargés de proposer à la clientèle de nouvelles offres et services, ce qui peut être considéré comme un point d'inflexion dans l'évolution du métier, davantage tourné vers le commercial.

\section{À l'origine, une logique de service public}

Les agents d'accueil sont tournés vers le service à l'usager dans une logique de service public. Leur éthique professionnelle est centrée sur la satisfaction de l'usager avec qui ils ont une relation de service directe. Cette posture doit donc être reliée au métier exercé par les agents, qui lui aussi a connu des évolutions : mutateur-codeur, secrétaires etc.; le contact avec le public a constitué une nouveauté dans leur vie professionnelle. Il y a eu un apprentissage et une qualification largement soutenus par le collectif de travail et leurs propres efforts à travers les formations qu'ils ont effectuées (comptabilité, bureautique...). Pourtant, nombre de conseillers ne parleront pas tant de leur métier, car ils n'ont pas le sentiment de détenir un vrai métier, que de la dimension sociale qui les motive dans le cadre du service public. C'est de l'expérience en ce domaine qu'ils tirent, selon leurs dires, leurs compétences, leur technicité. C'est sur le tas que s'élabore le sentiment d'appartenance et que se diffusent les valeurs du service public. Les termes « organisation» et «collectifs de travail efficaces » reviennent souvent dans les entretiens.

\section{Des activités de front et de back office}

En périodes de fortes pointes d'activité, les agents se mobilisent pour répondre aux appels avec le maximum de postes disponibles; mais lorsque dans la journée le nombre d'appels diminue, une part importante des agents présents se consacre alors au suivi des comptes, de la facturation, des contentieux... Reste à l'accueil téléphonique le nombre de personnes nécessaires pour assurer ce que sur les plateaux on nomme « la base ». Si le nombre d'appels augmente, ils basculent vers les agents qui sont en position de «débordement». Ces derniers doivent alors délaisser les tâches en cours pour répondre aux appels. Il y a ainsi un va-et-vient permanent entre les activités de «front office » et de «back office ». L'activité téléphonique est néanmoins prioritaire: elle occupe plus de $70 \%$ du temps d'affectation.

La rotation des postes entraine un déplacement des agents sur deux lieux distincts selon le flux des clients : la pièce en « back office » où sont traités les dossiers en attente, et les salles de réception téléphonique qui constituent le centre d'appels proprement dit. La polyvalence ne se traduit pas par plus de connaissances des processus, mais par l'obligation de réaliser des tâches supplémentaires, déconnectées des activités du «front », elles aussi fortement rationalisées ${ }^{5}$.

\section{Un changement de paradigme}

En 1990, le changement d'appellation de la branche Services (qui intègre PHONING), s'accompagne d'une refonte substantielle de l'organisation, notamment d'une concentration des anciennes agences locales en centre d'appels et d'une spécialisation du travail en fonction du type de clientèle ( $c f$. infra). Deux arguments sont convoqués pour justifier ce remaniement: l'augmentation des exigences clients et l'ouverture du marché européen avec la mise en concurrence avec d'autres formes d'énergie. Cette stratégie se confirme ces dernières années avec le projet d'ouverture du capital de l'entreprise et ses corollaires en termes de rentabilité et de productivité.

L'enjeu de ces réformes menées au nom du client est souvent posé par l'encadrement en termes d'adhé-

\footnotetext{
${ }^{5}$ Le back-office concentre la partie du métier qui requiert une plus grande technicité. Or cette technicité n'est pas répartie entre tous les travailleurs de la même façon. Les salariées affectées exclusivement au back-office prennent en charge les dossiers les plus complexes (impayés, réclamations dont la facture remonte à plusieurs mois, procédures judiciaires, etc.). Elles s'occupent également de tous les appels «en sortie» depuis leurs bureaux personnels. Les travailleurs plurifonctionnels, ceux qui réalisent des activités de back-office pendant leur temps de position « débordement ", sont affectés à des tâches plus simples. La plupart du temps, ils vérifient qu'il n'y a pas eu d'erreur informatique lors de l'établissement des factures, contrôlent un par un les relevés du compteur des clients dont la facture serait trop élevée, envoient les courriers standards, etc.
} 
sion des travailleurs à la nouvelle logique de l'entreprise, aux principes inclus dans son «tournant commercial » (Wiewiorka, Trinh, 1986). Dans le cas qui nous occupe, ces réformes se heurtent à une tradition professionnelle qui met en valeur le service public et le principe d'universalité du service rendu. Dans une poussée rationalisatrice, les pratiques non conformes des agents sont stigmatisées par l'encadrement comme des résistances au changement imprégnées de motivations archaïques. En revanche, les salariés interviewés ne cessent de revendiquer leur souci du service rendu au client. Ils développent alors une conception de la qualité et du service rendu qui les conduit à adopter des attitudes non fonctionnelles au regard des exigences de l'encadrement. Pour les salariés, la restructuration productive menace les valeurs autour desquelles ils ont constitué leur identité, leur éthique et leur autonomie professionnelle.

Dans ce cadre, la réorganisation de l'accueil et de la relation de service en centre d'appels et une redéfinition de la politique de gestion du personnel s'avèrent décisives pour moderniser l'entreprise et réussir l'adaptation du personnel. De ce point de vue, nous considérons les centres d'appels comme une fin en soi, en tant que dispositif permettant la relation immédiate avec le public et le contrôle de la clientèle et des marchés, et comme un moyen pour concrétiser les stratégies entrepreneuriales de modernisation selon les principes inscrits dans le « tournant commercial», rentabilité et productivité, par les opportunités qu'ils offrent en termes de flexibilisation et de rationalisation des pratiques professionnelles.

\section{LES CENTRES D'APPELS : LES USINES DU FUTUR?}

Certains auteurs ont signalé la diversité des situations de travail dans les centres d'appels, en fonction par exemple du secteur d'activité, de l'objectif de l'opération, de leur situation juridique par rapport à la société-mère (Cossalter, 2005), des politiques de gestion pratiquées, du type de rapports professionnels existants ou même du type d'implantation syndical (Lechat et Delaunay, 2003). Si cette diversité de situations observables en centre d'appels convient d'être rappelée, il existe néanmoins des éléments communs à tous les centres d'appels. En premier lieu, l'existence d'une rationalisation plus ou moins importante de l'activité et des échanges avec le public, autant en termes quantitatifs que qualitatifs. En second lieu, du point de vue subjectif, la nature même d'une activité qui, dans tous les centres d'appels, repose à la fois sur l'adaptation subjective à cette rationalisation et sur le contrôle orienté de l'émotivité.

\section{Encadré 2 \\ Le centre d'appels « PHONING »}

Chez PHONING, la création du centre d'appels s'accompagne d'une réorganisation des activités d'accueil et de gestion de la clientèle. L'organisation de l'accueil en centre d'appels suppose à la fois un regroupement des agences en réseau, et une segmentation de l'activité selon le type de clientèle. La rationalisation du travail sera plus ou moins poussée en fonction du type de clientèle. Sur les plateaux d'accueil " grand public » comme PHONING, cette rationalisation suppose une organisation du temps de travail en cycles courts, des scripts à suivre scrupuleusement et en même temps des objectifs en termes de services à placer.

Les plateaux d'accueil " professionnel » (les Centres de relation avec la clientèle-CRC), en revanche, fournissent aux usagers des renseignements plus précis, plus techniques aussi, par rapport à leurs installations et à leurs comptes : en général, les salariés de ces unités de production prennent en charge toutes les demandes dont l'objet échappe aux scripts téléphoniques des plateaux " grand public ». Dans ces unités de production, la durée moyenne d'un appel peut être d'une demi-heure. Enfin, nous retrouvons également des centres d'appels en sous-traitance, composés d'une main-d'œuvre sous-payée et déqualifiée, précaire. Ces centres d'appels prennent en charge les appels téléphoniques la nuit et le dimanche. 


\section{Une rationalisation industrielle du travail}

Chez PHONING, le travail est un travail d'exécution, caractérisé par un contrôle hiérarchique strict et par une forte dépendance technologique. Le contrôle hiérarchique s'effectue au travers du système informatique, grâce à un logiciel qui enregistre des informations précises décrivant les résultats individuels et collectifs de l'unité de production. Ce programme permet au superviseur $(n+1)$ de suivre en temps réel les activités et les résultats d'un nombre restreint de télé-conseillers (douze chez PHONING) : nombre d'appels réalisés ou reçus, nombre de services placés, durée des appels ou du temps de pause de chaque salarié, etc.

Le contrôle du rythme de production s'effectue à travers les dispositifs techniques de contrôle (Edwards, 1979). L'introduction d'autocommutateurs permet la gestion et la distribution automatique des appels téléphoniques entre les différents postes de travail et entre les groupes d'opérateurs auxquels correspond une file d'attente virtuelle: lorsqu'un appel se termine, le suivant (en position d'attente) bascule vers le poste qui s'est libéré. Les autocommutateurs fonctionnent comme des ordinateurs : grâce à eux, on programme par exemple le temps d'attente entre deux appels.

Le système technique intervient également dans la détermination du rythme et du contenu du travail au cours de l'appel téléphonique, comme dispositif de normalisation: par exemple, certaines fenêtres du logiciel ne sont accessibles qu'une fois la fenêtre précédente dûment remplie. Le logiciel est conçu pour assurer l'efficacité du système technique ainsi que sa propre auto-alimentation. La communication du téléconseiller avec le client est constamment perturbée par cette intromission du système technique, et les interruptions du salarié par l'usager sont très nombreuses :

«Client : bonjour madame, je vous appelle à propos d'une facture de $X \grave{a}$ une adresse qui n'existe plus puisque nous n'y habitons plus et pourtant cela fait au moins trois mois que j'ai signalé que nous n'habitions plus là... »

Téléopératrice : "pouvez-vous me donner vos références s'il vous plaît?"

Client : «Pfff (soupir) Euhh oui...»

Le travail des télé-conseillers est soumis à un impératif de rentabilité autour des critères comme le nombre d'appels à traiter, le nombre d'appels en attente tolérés, les temps moyens, le nombre de contacts réussis. Ces critères sont affichés sur des panneaux électroniques ou sur les écrans des ordinateurs; ils composent les statistiques des performances individuelles et collectives et encadrent la durée et le contenu des échanges avec le public.

L'impératif de rentabilité passe par le contrôle des interactions téléphoniques. La volonté de contrôle induit une rationalisation de l'échange verbal et de son amplitude. La rationalisation doit permettre au salarié de diriger la discussion et de contrôler sa durée. Du point de vue de la gestion, l'objectif est de faire en sorte que le salarié réponde à un maximum d'appels, en un minimum de temps, et tout cela dans le respect des contenus prescrits de l'échange.

Le parti-pris de cette forme d'organisation du travail d'inspiration taylorienne est de considérer la diversité des situations comme réductible à un nombre restreint de cas typiques normalisés, au point que l'on peut en confier la gestion à des salariés sans qualifications particulières dans le domaine de la vente ou des services concernés.

Le télé-conseiller pose un certain nombre de questions qui s'affichent à l'écran et il tape chaque fois la réponse apportée. La transparence du système oblige l'agent à la coopération puisque les termes de l'échange sont définis par le logiciel : une sortie du circuit serait immédiatement repérée.

Enfin, le logiciel contient des argumentaires commerciaux, ou des réponses-types à des questions recensées auparavant, des mots-clés, etc., pour vendre les différents services offerts par l'entreprise. Cela accentue l'aspect commercial de l'échange fondé par définition sur la persuasion du client. L'exemple présenté ci-après est révélateur. Notons que les gestionnaires prévoient un ensemble de situations possibles et de réponses argumentées face à un refus éventuel du client de souscrire à un service payant :

Scénario: le client emménage dans un logement équipé au gaz naturel :

1. Conseil et proposition de service d'énergie :

1.a) électricité $\rightarrow$ usages $\rightarrow$ orientation vers le Conseiller X

1.b) gaz naturel $\rightarrow$ usages

1.b.1) Diagnostic payant ou,

1.b.2) Diagnostic non payant si installation au gaz inactive depuis 6 mois. 
1.b.1) La proposition de service : Diagnostic qualité OFFRE PAYANTE

Agent accueil : "Vous devriez profiter de votre emménagement pour faire vérifier la qualité de votre installation gaz naturel Pour cela, nous vous proposons le service Diagnostic qualité $X$. »

(Plusieurs phrases-clés)

Client : «Oui, mais 30 euros c'est cher, je n'ai pas les moyens!»

Agent d'accueil : "Le coût réel de cette intervention est de 20 euros, cela correspond au coût de la prestation de l'expert indépendant, l'entreprise ne souhaitant pas être juge et partie. Nous contribuons à hauteur de 16 euros au financement de cette prestation. Votre participation de 14 euros est un prix raisonnable pour votre tranquillité et la sécurité de votre famille et de votre entourage. Il est important de savoir si votre installation est en bon état de fonctionnement. »

Codification des questions et des réponses :

1.b.1.a) pas OK tout de suite $\rightarrow$ mailing relance

1.b.1.b) traitement de la demande $\rightarrow$ prise de RDV sur le logciel OPTIMIA pour intervention du technicien.

\section{Un double travail sur soi : s'adapter et contrôler son émotivité}

L'activité des salariés est à la fois routinière et stressante. Ils mobilisent d'abord des routines linguistiques qui, faisant l'objet de formatages et de contrôle, deviennent, du «prêt à parler» (Boutet, 2001). Dès lors, pour mettre en mots le rapport au travail, les conseillers de PHONING utilisent des comparaisons, des archétypes qui renvoient à un travail dévalorisé, répétitif: «Je pense avoir des capacités pour réaliser un travail bien meilleur que celui que je fais actuellement. Dans le plateau, il m'arrive d'activer 'le pilote automatique' [...]» (Conseillère de clientèle, 23 ans, bac commercial, 2 ans d'ancienneté) Ou encore : «N'importe qui pourrait faire ce travail, on n'a pas besoin d'un grand degré d'intelligence pour répéter toujours la même chose comme un perroquet. » (Conseiller de clientèle, 23 ans, bac commercial, 18 mois d'ancienneté)

Pourtant, si le caractère taylorien de l'activité est récurrent dans les entretiens, il peine à rendre compte de la nature du travail des télé-conseillers dans les centres d'appels. Chez PHONING, les dispositifs de taylorisation de l'activité vocale (Buscatto, 2002) se réfèrent fondamentalement à l'aspect quantitatif de l'activité. Bien que cet aspect soit décisif et prioritaire en situation de travail, il ne suffit pas à définir le travail. C'est aussi un travail d'écoute, d'estime, de proposition, de séduction de la personne qui est au bout du fil. C'est un travail dont la qualité dépend directement de la manière de travailler, un travail soumis immédiatement au jugement du client.

En situation de travail, les télé-conseillers ne doivent pas se contenter de répéter un même script. Ils doivent donner au client l'impression qu'ils l'écoutent; adapter la conduite de la discussion au type de client ; faire en sorte que le client se sente reconnu dans la relation téléphonique (Cousin, 2002), notamment par le ton de la voix qui doit être approprié à chaque situation En somme, la subjectivité du travailleur, son attitude, ses émotions, sont indissociables du produit.

Cela induit un nouveau décalage entre l'identification du type d'attitude requise pour chaque situation, la manière dont la direction entend utiliser ces compétences sociales et la façon dont les salariés les utilisent ; en effet, les registres qui motivent les uns et les autres à s'impliquer dans le développement de l'activité ne coïncident pas nécessairement.

Pour l'entreprise, les travailleurs doivent mobiliser leurs aptitudes relationnelles mais pas de n'importe quelle manière. Les dispositifs d'entraînement comme le coaching servent en réalité à aborder des aspects spécifiques du travail non complètement prescriptibles (Calderon, 2005a). L'objectif étant de faire adopter aux télé-conseillers une logique de comportement conforme aux objectifs de l'entreprise. Néanmoins, l'introduction de l'enjeu économique au cœur même de l'action percute la finalité du travail des salariés des plateaux de clientèle, délite le compromis historique et fragilise les anciennes formes des rapports de travail et au travail. Dans ce nouveau contexte, les salariés se repositionnent.

\section{FORMATER LA SUBJECTIVITÉ DES SALARIÉS}

L'encadrement utilise les processus de recrutement pour repérer les individus dotés de compétences sociales appropriées pour la production de services. Chez PHONING, l'attitude n'est plus une partie 
importante du travail mais le travail en soi. Les salariés sont recrutés pour produire des unités " d'attitude ». Pourtant, les dispositifs de sélection et de recrutement peinent face à la complexité du processus de production. Ce travail est fondamentalement réalisé via les dispositifs d'entrainements qui visent à apprendre à « construire la relation » avec le client à partir de paramètres bien définis.

Sur le plateau, il y a plusieurs types de formations informelles, ou coaching ${ }^{6}$, selon qu'elles concernent les processus et produits de travail ou les techniques de communication, ou qu'elles sont dispensées par des responsables de formation ou par l'encadrement de proximité. La fréquence est d'un coaching par mois et par agent ; ces coaching ont lieu dans le bureau des responsables de formation, ou sur le poste de travail.

Le coaching vise notamment à ce que les salariés soient en mesure de contrôler la conversation avec le client, de façon à ce qu'aucun des éléments de la conversationtype ne leur échappe. Il s'agit d'intérioriser les mécanismes de contrôle des éléments aléatoires de la conversation qui peuvent nuire à son développement standard. Avoir le plan en mémoire, donner la priorité à ce plan face à la situation vécue permettrait de faire abstraction de la discussion et du climat qu'elle peut générer, parfois violent, pour atteindre un à un les objectifs : répondre à la demande du client et proposer des services, en un minimum de temps.

Ainsi, la prestation standard ne se définit pas seulement en termes de résultats (la résolution du problème et la fidélisation du client), mais en termes de prescription des éléments intermédiaires de la discussion : les résultats intermédiaires, durée, séquences que doit respecter chaque appel. Dans ce sens, elle réaffirme la centralité du système technique. Globalement, elle consiste à éviter que les salariés fonctionnent à leur façon, ce qui ajouterait un problème de gestion, et donc politique, pour le management : comment savoir si la norme n'est pas

\footnotetext{
${ }^{6}$ La Société française de coaching (association de professionnels) définit le coaching comme «l'accompagnement des personnes ou des équipes pour le développement de leurs potentiels et savoirfaire dans le cadre d'objectifs professionnels ». Le cadre conceptuel, revendiqué par les professionnels, dans lequel s'ancrent les pratiques de coaching, trouve ses fondements dans le constructivisme de l'École de Palo Alto et la PNL (programmation neurolinguistique) (voir www.sfcoach.org).
}

respectée parce qu'elle ne peut pas l'être ou parce que les salariés ne veulent pas la respecter? Le management se trouverait confronté à la fois à des situations où les modalités de conduite de la conversation sont réinterprétées en fonction du plan, et à des situations où l'expérience d'un travailleur ou d'une équipe les conduit à ignorer ou à refuser les valeurs prescrites. Chez PHONING, la direction ne semble pas être disposée à accepter cette perte de contrôle sur les processus de travail. Au contraire, «le coaching n'est rien d'autre que le moyen que nous avons pour faire passer la politique de qualité de l'entreprise» (Manager Centre). Ainsi, la pression exercée sur les salariés finirait par leur faire intégrer les objectifs du management : "Il faut que ça rentre dans les mours, que ça devienne un automatisme. Les principes clés du plan doivent devenir des automatismes. » (Manager Centre) Ce caractère automatisant n'échappe pas aux salariés rencontrés : "Leur objectif est très clair : que nous ne soyons pas des personnes, que nous répondions comme des répondeurs automatiques, comme des robots. Le coaching approfondit cette dynamique: oublier qui tu es et fonctionner à partir d'expressions toutes faites. " (Téléconseillère, 43 ans, 22 ans d'ancienneté)

Ce premier type de coaching souligne le caractère automatisant du travail caractérisé par le dirigisme en matière de respect du prescrit. La deuxième dimension du coaching insiste en revanche sur la réactivité au cours de la conversation avec le client. Plusieurs exercices de coaching se focalisent sur la construction d'un comportement standard à adopter par les télé-conseillers. D'autres portent sur les capacités à influencer le contexte émotionnel par la modulation de la voix. "La phrase: 'le sourire s'entend' n'est pas une stupidité. Au contraire, c'est quelque chose de fondamental. » (Responsable de formation) Cette même responsable de formation nous confiait un peu plus tard au cours de l'entretien: "Bien communiquer, ce n'est que séduire qui t'écoute.» Les techniques de séduction occupent une place primordiale dans la formation des salariés: "influencer le contexte émotionnel» et "produire des sentiments contrastés », et ce par la modulation de la voix, faute de contact visuel avec le client.

Un des exercices que nous avons pu observer consiste à montrer aux salariés le pouvoir de la simulation. Pendant l'exercice, une partie des stagiaires circulent 
avec les encadrants dans la salle alors que les autres, par couples, simulent des discussions avec le client. Ceux qui observent sont censés prendre des notes, et avec l'aide des encadrants, ils se questionnent sur la meilleure façon de construire la discussion en fonction des circonstances. Selon un encadrant ayant participé à cet exercice, les stagiaires s'amusent à voir comment, par un effet de mimétisme, certains agents finissent par imiter des gestes et expressions d'autres agents, ce qui, à son avis, "constitue la meilleure façon d'apprendre ».

Par le coaching, il s'agit d'apprendre à « construire la relation » au travers de techniques pré-établies de contrôle de la conversation, y compris de techniques de séduction. Cette idée que la relation se « construit» est capitale dans le processus. Cependant, si lors des coaching, l'accent est mis sur le rapport au client et la qualité de la discussion, en situation de travail, c'est le nombre d'appels enregistrés qui prime. Autrement dit, une certaine « attitude » est pré-requise pour le bon déroulement des activités, mais en même temps elle est modulée par les dispositifs mis en place. Le coaching est alors utilisé comme un dispositif de contrôle supplémentaire.

En situation de travail, les salariés doivent contrôler leurs émotions (Hochschild, 1983), pour faire abstraction de leurs sentiments et les adapter aux normes de l'entreprise. Il s'agit de créer un personnage au travail. Le coaching fixe le profil-type et facilite l'adaptation à ce profil-type, moyennant le suivi systématique et méthodique du processus de professionnalisation de chacun. Certains salariés l'ont illustré sans équivoque : "Ici sur le plateau, il y plusieurs personnalités, mais ils essayent de les modéliser toutes à partir d'un modèle unique, le commercial X. Et ils poussent et poussent pour que nous soyons tous pareils. » (Conseillère, 35 ans, 10 ans d'ancienneté)

\section{AU COEUR DE L'ACTIVITÉ : LA TENSION ENTRE INTERACTION ET RATIONALISATION}

Les salariés des centres d'appels savent que le coaching vise à rationaliser des aspects de leur subjectivité pour les inciter à se dédoubler, à jouer un rôle. On attend d'eux qu'ils soient réactifs, disponibles, flexibles. Du point de vue subjectif, on exige qu'ils acceptent les critères qui régissent leur activité, ainsi qu'une certaine logique de comportement (le commercial souriant).

L'appel à l'implication, pourtant, ne remplace pas mais accompagne cette normalisation, cette nouvelle technicisation des process de travail qui s'inscrit ainsi dans les critères de rentabilité et de productivité de l'entreprise. La notion de combinatoire de modalités de contrôle rend compte de ces processus : dans les centres d'appels, le salarié en tant que sujet se trouve engagé dans l'activité, coupé de son collectif de travail et conditionné par la juxtaposition d'impératifs qualitatifs et quantitatifs qu'il doit concilier.

Dans ce cadre, l'objectif de dispositifs comme le coaching n'est autre que de faire adopter aux téléconseillers une logique de comportement conforme aux objectifs de rentabilité de l'entreprise. Pourtant, ces objectifs sont souvent en contradiction avec la "qualité de la prestation », ou la notion même de service rendu à l'usager, telle qu'elle est comprise par les salariés : «Nous sommes face au client et sommes obligés de respecter les objectifs de rentabilité de l'entreprise, il n'y a pas moyen de s'y opposer [...] On n'est plus dans une logique de service public et on pourrait même se demander si on est dans une logique de service tout court. » (conseillère de clientèle, 23 ans d'ancienneté)

«Le plus dur, ce n'est pas seulement le type de travail qu'on fait ici, fatigant, ennuyeux, stressant... le plus dur, c'est de devoir sourire tout le temps; le plus dur, c'est de devoir vendre des services alors que ce ne devrait pas être notre rôle, le plus dur sans doute, c'est de devoir porter la stratégie de l'entreprise alors que nous on est opposés à cette stratégie, alors que nous croyons encore à notre rôle de service public. » (conseiller de clientèle, 17 ans d'ancienneté)

La subjectivité des salariés de ce type de platesformes téléphoniques se trouve doublement atteinte : d'une part, parce que l'activité est de nature hétéronome, répétitive et stressante et ne laisse guère de place à l'autonomie et à la créativité. De ce point de vue, la comparaison avec les lignes de montage traditionnelles est fortement heuristique puisque, dans les deux cas, le système technique ne considère pas les travailleurs comme des êtres humains ayant des motivations et des intérêts propres mais, au contraire, suscite chez eux le sentiment d'être programmés. «Robots», «répondeurs automatiques», comme 
l'illustrent les termes utilisés par les conseillers de clientèle interviewés. Mais la subjectivité des salariés se trouve également atteinte puisque, en situation de travail, les salariés du centre d'appels sont contraints d'oublier qui ils sont véritablement, quelles sont leurs émotions et leurs motivations, leur histoire, leur éthique, de manière à jouer un personnage, le commercial souriant. L'orientation patronale qui vise à associer les salariés au projet de l'entreprise implique pourtant d'importants bouleversements, notamment que les salariés rompent avec l'ancien projet communément partagé par le personnel de l'entreprise; c'est-à-dire, au fond, qu'ils rejettent une appartenance, une vie liée à une culture de service public. Dans chaque acte de travail, cette nouvelle dimension dessine une ligne de tension entre les salariés et leur direction : la question de la finalité du travail.

Bien sûr, l'intensité de l'enjeu sera différente selon les trajectoires des salariés. La dimension générationnelle joue ici un rôle qu'on doit néanmoins relativiser. Certes, les salariés, notamment les anciens, doivent renoncer à une histoire commune attachée à la défense des valeurs du service public, ce qui est vécu comme une véritable atteinte à leur identité. Quant aux plus jeunes, ils doivent entrer en relation personnalisée avec autrui tout en étant eux aussi dans l'incapacité de la gérer selon leur propre sensibilité, qu'ils se reconnaissent ou pas dans une culture de service public. Ils sont ainsi personnellement engagés vis-àvis d'autrui avec qui ils peuvent éventuellement s'identifier. Pour certains, cela engendre une véritable souffrance, un sentiment de ne plus se reconnaître dans l'activité; chez d'autres prévaut un sentiment d'impuissance face aux besoins d'autrui, qui peut se trouver dans des situations de détresse sociale et émotionnelle. Dans tous les cas, le temps au travail est pesant car fatiguant, stressant, répétitif, mais aussi source d'impuissance face aux contraintes externes.

Il faut noter que le taux d'absentéisme frôle les 40 \% chez PHONING. Le départ en maladie se banalise : une jeune télé-conseillère répondait ainsi à une question relative à la fréquence de prise de médicaments : «non, je ne prends pas de médicaments parce que ça fait peu de temps que je suis dans la boîte. " (conseillère de clientèle, 21 ans, 6 mois d'ancienneté) La prise de médicaments chez PHONING concerne $80 \%$ du personnel.

\section{Deux types de pratiques d'adaptation/résistance}

Dans ce contexte, nous avons identifié deux types de pratiques d'adaptation/résistance: des pratiques de « mise en veille» et des pratiques "d'implication éthique dans l'activité », qui ne sont pas contradictoires. Il serait même réducteur de penser que certains salariés privilégient une pratique plutôt que l'autre. Au contraire, les salariés peuvent changer de registre en l'espace de deux appels. Il faudrait donc tenter de les appréhender dans un continuum qui manifeste le même enjeu : se sentir ou non reconnu dans son travail, la lutte du salarié pour faire valoir la dimension éthique de son implication au travail face à une orientation stratégique qui lui est imposée au quotidien.

Le premier type de pratique (l'adaptation) correspond aux situations dans lesquelles les conseillers donnent l'impression de respecter les modalités (objectives et subjectives) de conduite de la conversation. Chacun a pu faire l'expérience de ce genre d'appel téléphonique en provenance d'un centre d'appels au cours duquel l'opérateur répète sur le même ton, comme exagérant la norme de séduction requise, les scripts qui lui sont imposés. Ces attitudes de "mise en veille» ou d'application d'une "émotivité de surface » (Taylor et Bain, 2002) correspondent, dans le contexte des centres d'appels, à de véritables lignes de fuite dans la représentation du personnage, dans la simulation du « commercial souriant», de la part des salariés, comme s'il existait une sorte de démission de soi dans la réalisation de son activité. C'est alors la dimension adaptative qui prévaut, mais elle ne permet pas aux salariés de se sentir reconnus dans leur activité et « la séparation entre force de travail et sens de l'activité est maximal» (Lara, 2004, p. 46). Ces types de pratiques de «mise en veille » peuvent constituer une ressource pour résister à la pénibilité de l'activité, au stress, à l'agressivité du client (Jeantet, 2001). Mais elles finissent par être pénibles pour les salariés et dévalorisantes.

Nous avons repéré un second type de pratiques de résistance. Les salariés s'emparent alors de leur activité et mobilisent certaines de leurs aptitudes individuelles et collectives, mais à partir de registres divergents avec la logique de productivité et de rentabilité. Chez PHONING, cette réappropriation du 
travail et de sa finalité par des téléconseillers s'inscrit dans l'histoire des collectifs de travail, soucieux de leur autonomie opérationnelle, de leur qualification technique et relationnelle et de leur spécificité en tant que porteurs d'une mission de service public?

En situation de travail, les salariés s'informent des problèmes qui apparaissent au fil des journées, ou sur la meilleure manière de répondre à un questionnement non prévu dans les scripts, et ils le font dans une logique de service rendu à l'usager. Ce faisant, les salariés constituent un ensemble de qualifications informelles qu'ils utilisent pour éviter d'orienter les appels vers les CRC, ce qui laisse penser que les opérateurs cherchent à valoriser leur activité auprès du collectif de travail et à leurs propres yeux. Des espaces de liberté se constituent ainsi, éloignés de l'optique de l'entreprise, et peuvent donner lieu à des confrontations plus ou moins explicites avec l'encadrement en même temps qu'ils renforcent les collectifs de travail autour de ces mêmes valeurs.

Plusieurs exemples peuvent être évoqués ici. Une pratique récurrente est celle de la personnalisation qui consiste à donner au client son numéro de poste. Cette pratique contredit les indications de la hiérarchie à ce sujet, et les notes informatives et les réunions organisées par la hiérarchie pour l'éradiquer sont assez courantes. Nous avons pu assister à une de ces réunions : les opérateurs objectaient que les situations devaient être examinées au cas par cas, en fonction des besoins de chaque client, alors que la direction soulignait que la pratique de la personnalisation perturbait le bon fonctionnement du réseau et la traçabilité des clients. Elle rétorquait également que ce type de pratiques tendait à occulter le nombre total d'appels. Mais c'est oublier que la mission des télé-conseillers chez PHONING est une mission de contact avec l'usager. Cela s'est toujours déroulé dans le respect des procédures, des lois, des règles, mais avec la possibilité de les interpréter au mieux

\footnotetext{
${ }^{7}$ Nous utilisons ici une définition du collectif de travail qui accorde une place prépondérante à la dimension éthique de l'implication au travail. Les règles éthiques distinguent ce qu'il est juste de faire de ce qui ne l'est pas. Si les salariés n'arrivent pas à se mettre d'accord sur les valeurs qui régissent leur activité, les collectifs s'effondrent inexorablement. Cette démarche délibérative est devenue aujourd'hui problématique, mais en même temps, elle peut donner naissance à des collectifs de travail renouvelés, éloignés de la rationalité essentiellement marchande de l'entreprise.
}

dans l'intérêt du client, d'assouplir certaines conditions ou délais, par exemple les délais de règlement des factures ${ }^{8}$. Aujourd'hui encore, la plupart des téléconseillers refusent par exemple de proposer des services payants à des clients qui éprouvent des difficultés à régler leurs factures: "c'est immoral de nous obliger à proposer des services payants à quelqu'un qui n'arrive même pas à payer le service minimum. » (Conseillère de clientèle, 27 ans, 5 ans d'ancienneté)

De même, les télé-conseillers outrepassent leurs attributions formelles lorsqu'ils décident de prendre partie pour un type de service plutôt qu'un autre, par exemple le service d'une compagnie qu'ils considèrent meilleur: "Par exemple, parfois on nous demande si c'est mieux (tel ou tel type d'énergie). La direction nous interdit de répondre à ce type de question et d'orienter le client vers les CRC des deux compagnies puisqu'elles sont séparées depuis la privatisation de la compagnie, et nous ne devons pas en principe prendre partie. Mais c'est absurde, puisque c'est évident qu'un type d'énergie est meilleur, il est plus économique et plus propre et donc on n'hésite pas à proposer au client de le choisir plutôt que l'autre. Et demande à qui tu voudras : on fait tous pareil sur le plateau!" (Conseillère de clientèle, 32 ans, 9 ans d'ancienneté)

Cette dimension éthique de l'activité est importante car elle fournit de la consistance et une teneur particulière à l'identité élaborée autour de la notion de service public. Elle confère tout son intérêt à l'activité. Les valeurs du service public se dénotent encore dans la façon d'instruire un dossier, aux dires des interviewés. La réponse donnée est aussi la marque personnelle de l'agent ou du collectif qui la produit. Le salarié s'investit autant comme individu que comme membre d'un collectif qui fonctionne selon

\footnotetext{
${ }^{8}$ La défense de l'autonomie professionnelle est un élément fondamental de l'éthique professionnelle des salariés. Les salariés ont disposé d'une marge de manœuvre importante dans la gestion des dossiers, et plus particulièrement dans l'attribution des délais de paiement. Grâce à cette autonomie, les agents pouvaient proposer des solutions et ne pas rester impuissants face aux problèmes du client, tout en garantissant le respect des procédures. "Question: Et qu'est-ce qui se passe quand vous avez des injonctions contradictoires à gérer? Réponse: On discute entre nous pour trouver une solution convenable pour l'usager » (Conseillère de clientèle, 31 ans, 10 ans d'ancienneté).
} 
des règles sociales et éthiques collectivement construites et comme représentant de son entreprise. C'est pourquoi, malgré la démotivation rampante, la sollicitude due à l'usager oblige à agir : "On se met aussi à la place de certains clients. Parce que c'est aussi un peu une conscience quand même. Le client ça peut être vous, ça peut être moi. » (conseiller de clientèle, 31 ans, 10 ans d'ancienneté) Ou encore : «c'est notre mission. Et contrairement à ce que peut penser notre direction, on a ça ancré au fond de nous, et on n'a pas besoin de nous demander quelque chose. On sait ce qu'on a à faire. » (conseillère de clientèle, 47 ans, 23 ans d'ancienneté)

La gestion au nom du client suscite moins, pour l'instant peut-être, une opposition d'ordre culturel que des conduites visant à préserver une certaine autonomie professionnelle. En ce sens, les salariés n'opposent pas référence au service public et référence au client mais contestent la conception managériale du service au client au nom de leurs propres valeurs. Les salariés revendiquent un service au client de qualité (qualité de la relation téléphonique, du conseil ou du traitement du dossier), au-delà des exigences en termes de rentabilité, qu'ils remettent en cause. Cette valorisation de la qualité du service rendu permet de continuer à faire vivre les anciennes valeurs et de se constituer un certain quant-à-soi par rapport à l'organisation du travail et à son emprise. Elle permet aussi, comme par le passé, d'affirmer la qualité des salariés et des collectifs de travail et d'en appeler à la reconnaissance d'une conscience professionnelle et d'une éthique que l'organisation du travail cherche à occulter.

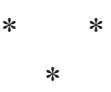

Les centres d'appels sont le lieu d'une intéressante contradiction, révélatrice d'évolutions plus larges dans le monde du travail. Rarement, dans l'histoire de la rationalisation du travail, le «facteur humain » a (officiellement) occupé une place si décisive dans la rentabilité : ce qui compte aujourd'hui, ce n'est pas seulement le temps objectif, c'est-à-dire le travail objectivé, la rationalisation des temps et des gestes de travail, mais le temps subjectif de production, le sens de l'implication des salariés, les valeurs qui soustendent cette implication. À la rivalité taylorienne (entre dirigeants et salariés) concernant l'usage du premier, devrait se substituer un usage conjoint du second pour parvenir à des fins compatibles. Mais la radicalité du changement est limitée, du moins dans les centres d'appels, par deux facteurs : d'une part, la prégnance des normes temporelles et du cadre prescriptif témoigne de la vigueur du modèle taylorien dans ces organisations; d'autre part, les objectifs de l'entreprise, la rentabilité et la productivité du dispositif ne sont pas négociables puisqu'ils sont inscrits dans l'organisation du travail et de la production, de même qu'une définition éthique de la relation avec le public est imposée aux salariés.

Les centres d'appels représentent ainsi l'évolution peut-être paradigmatique du taylorisme vers une nouvelle organisation de type néo-tayloriste qui requiert, à la différence des organisations strictement tayloriennes, une certaine implication de la part des travailleurs; cette implication étant modulée, nous l'avons montré, par les dispositifs de formation mis en place. Or, dès lors que le temps subjectif de production devient une préoccupation de la direction, cela crée une tension puisque la finalité même du travail devient un enjeu: pourquoi travaille-t-on? Pourquoi s'implique-t-on dans la recherche de nouvelles manières de procéder?

Une télé-conseillère de 31 ans déclarait ainsi : «On nous demande de mentir un peu, que ce n'est pas si grave, qu'on oublie ce qu'on pense véritablement, mais au final tous ces petits mensonges deviennent un grand mensonge et nous participons tous de ce grand mensonge. » (Conseillère de clientèle, 31 ans, 10 ans d'ancienneté)

Bien sûr, on pourrait considérer, en partant de la crainte exprimée par cette télé-conseillère, que le système fonctionne parce que eux, les télé-conseillers des centres d'appels, s'accommodent du mensonge, de l'injustice. Ils sont de ce point de vue «traversés de fait par la stratégie de l'entreprise », comme nous le confiait un autre d'entre eux. Pourtant, de manière également manifeste, le système fonctionne aussi parce que chacun lui oppose une résistance, parce que les salariés, loin de se comporter comme des rouages de ce même système qui ne les considère pas comme des personnes ayant des intérêts et des motivations propres, mettent en revanche un peu d'humanité dans sa logique monolithique. À cet égard, chacun jongle avec la norme prescrite et s'efforce de transformer les rapports sociaux qui pèsent sur lui en fonction de ses propres exigences éthiques. 
Les salariés prennent au sérieux les contradictions de leur activité, et ils ne sont pas prêts à tout pour parvenir aux objectifs prescrits. Et le départ en maladie de certains d'entre eux montre bien que ce qu'ils engagent dans l'activité est bien plus que la satisfaction d'objectifs nécessairement abstraits fixés par l'entreprise. Le cas de PHONING est particulièrement révélateur à cet égard: la direction refuse d'arbitrer entre la norme marchande et la norme sociale; elle demande simplement aux salariés de respecter un code de conduite fondé sur la rentabilité. Pourtant, les travailleurs doivent opérer cet arbitrage, car leur activité suppose une médiation entre exigences marchandes et exigences sociales, comme le respect de l'autre ou une certaine sincérité dans l'échange.
Dans le cas des plateaux de clientèle, le principal problème est que l'enjeu de la finalité du travail, la compétition pour le contrôle du sens accordé à l'activité entre les salariés et l'encadrement, semble pour l'instant circonscrit à l'individu, et n'est pas reconnu par la direction dans la plupart des cas. Les pratiques de travail basculent ainsi du strict respect des consignes à l'implication active dans les problèmes de l'usager. De la façon dont cette tension est ou sera résolue dans chaque centre d'appels dépendra le champ des possibles, l'avenir de cette profession: une profession dévalorisante, déshumanisante et répétitive pour les salariés ou, au contraire, une profession qui intègre la dimension qualitative de la relation, qui la place au cœur de l'organisation et avec elle les attentes des usagers et des salariés.

\section{Bibliographie}

Abecassis F., Roche P. (2001), Précarisation du travail et lien social. Des hommes en trop, Paris, L'Harmattan.

Bain P., Taylor P. (2002), "Entrapped by the Electronic Panopticon ? Worker Resistance in the Call Centre", New Technology, Work and Employment, 15(1), pp. 2-18.

Boltanski L., Chiapello E. (1999), Le nouvel esprit du capitalisme, Paris, Gallimard NRF.

Bonazzi G. (2002), Storia del pensiero organizzativo, Milan, FrancoAngeli.

Bono A. (2001), "Call Centers, ¿el trabajo del futuro? El caso de estrategias telefónicas S.A", Sociología del Trabajo, n 39 , pp. 3-31.

Boutet J. (2001), «Le travail devient-il intellectuel ? », Travailler, $\mathrm{n}^{\circ}$ 6, pp. 55-70.

Braverman H. (1974), Labour and monopoly capital, Montlhy Review Press, Londres.

Burawoy M. (1979), Manufacturing Consent, Chicago Press.
Buscatto M. (2002), « Les Centres d'appels, usines modernes? Les rationalisations paradoxales de la relation téléphonique », Sociologie du Travail, $\mathrm{n}^{\circ} 44$, pp. 99-107.

Calderon J. (2005a) « L'implication quotidienne dans un centre d'appel. Les nouvelles initiatives éducatives », Travailler, $\mathrm{n}^{\circ}$ 13, pp. 75-94.

Calderon J. (2005 b), « Les pratiques de résistance face à la manipulation de la subjectivité » Communication et organisation, $\mathrm{n}^{\circ} 28$, dossier « Coaching et Communication ».

Calderon J. (2006), "Repensar la cuestión de la resistencia en el trabajo o buscando al trabajador perdido. Un estudio de caso en el sector del telemárketing", Sociología del Trabajo, $\mathrm{n}^{\circ} 56$.

Callaghan G., Thompson P. (2002), “We recruit attitude: the selection and shaping of routine call centre labour", Journal of Management Studies, n 39:2, pp. 233-254.

Cossalter Ch. (2005), «La compression des temps. Quand les services financiers s'équipent en plateaux 
téléphoniques », in Linhart D. et Moutet A., Le temps nous est compté.

Cousin O. (2003), « Les ambivalences du travail. Les salariés peu qualifiés dans les centres d'appel», Sociologie du Travail, $\mathrm{n}^{\circ}$ 44(4), pp. 499-520.

Delaunay J.-C., Lechat L. (2003), Les Centres d'Appels : un secteur en clair-obscur, Paris, L'Harmattan.

Edwards R. (1979), Contested Terrain. The Transformation of the Workplace in the Twentieth Century, Londres, BasicBooks.

Flichy P., Zarifian P. (2003), « Les centres d'appels », Réseaux, $\mathrm{n}^{\circ} 114$.

Gadrey J. (2006), La productivité en question, Paris. Desclée de Brouwer.
Hochschild A. (1983), The Managed Heart: the Commercialization of Human Feeling. Berkeley. Univ. of California Press.

Jeantet A. (2001), La relation sociale de service, Thèse de sociologie, université Paris X-Nanterre.

Lara A. (2004), "El telemarketing en España : materiales para una cartografía del mundo del trabajo contemporáneo", Sociología del Trabajo, n 49, pp. 27-59.

Linhart D. (2004), La modernisation des entreprises, La Découverte, Paris.

Wiewiorka M., Trinh S. (1986), Le modèle EDF. Essai de sociologie des organisations, Paris, La Découverte.

\title{
Résumé
}

\section{Le travail face à la restructuration productive : le cas d'un centre d'appels}

\author{
José Calderón
}

Les centres d'appels cristallisent une série de tendances qui affectent le monde du travail dans son ensemble : ce ne sont plus seulement les temps et les gestes du travail qui sont objectivés et rationalisés. L'encadrement accorde de plus en plus d'importance à la subjectivité du salarié, c'est-à-dire aux valeurs engagées et mobilisées au travail et à sa finalité. Ainsi, le salarié doit faire un usage de son temps de travail et de ses compétences le plus efficace possible par rapport aux objectifs de l'entreprise. Si la subjectivité du salarié devient une préoccupation de l'encadrement, alors l'usage subjectif du temps de travail devient aussi un enjeu. Pour développer cette double hypothèse, nous nous appuyons sur les résultats d'une recherche par immersion réalisée dans un centre d'appels.

Mots clés

Centre d'appels, encadrement, temps de travail, organisation du travail

Journal of Economic Literature : J 53, J 81 